\title{
Prevención del consumo problemático de drogas en la escuela: estrategia de formación docente en Argentina utilizando TIC*
}

\author{
Ana Clara Camarotti ${ }^{1}$ \\ Ana Lía Kornblit ${ }^{2}$ \\ Pablo Francisco Di Leo ${ }^{3}$
}

\section{Introducción}

El presente trabajo tiene por propósito analizar los discursos de docentes participantes del curso virtual sobre Prevención del Consumo Problemático de Drogas, dictado en forma conjunta por el Ministerio de Educación de la Nación Argentina, el Instituto de Investigaciones Gino Germani de la Facultad de Ciencias Sociales de la Universidad de Buenos Aires y UNICEF Argentina. El objetivo general del curso virtual fue desarrollar un programa de capacitación docente, con alcance nacional, que suministre herramientas teóricas y prácticas para la prevención del consumo problemático de drogas en escenarios escolares.

El curso se estructuró en base a 6 ejes temáticos, los que conformaron 6 módulos que se dictaron con una frecuencia quincenal a lo largo de 3 meses ( 2 módulos por mes). Se previó el desarrollo de foros de intercambio a cargo de tutores para compartir dudas y reflexiones de los cursantes en base a las lecturas de los materiales.

En los módulos se examinaron las posturas de los autores más relevantes que han trabajado en cada uno de los temas abordados, estimulando en los cursantes el análisis crítico y la capacidad de síntesis.

El objetivo del trabajo aquí presentado es identificar los principales tipos de posiciones discursivas presentes en las intervenciones de docentes en los foros de los 6 módulos del curso. Con dicho fin, analizamos las participaciones de alrededor de 3000 docentes que cursaron durante 2010. Para el análisis del corpus seguimos los lineamientos generales de la teoría fundamentada, utilizando como auxiliar el software Atlas.ti. Aplicando los criterios de parsimonia-maximizar la comprensión de un fenómeno con el mínimo de conceptos posible - y de alcance - ampliar el campo de aplicación del análisis sin desligarse de la base empírica -, en diálogo con el estado del arte y el marco conceptual, identificamos las categorías emergentes de los discursos de los docentes en torno a consumos de drogas, jóvenes, adultos y escuela. A continuación, articulando las categorías emergentes, presentamos los dos tipos de posición discursiva identificados, a los que denominamos: Modelo negativizante-unidimensionaly Modelo reflexivo-multidimensional.

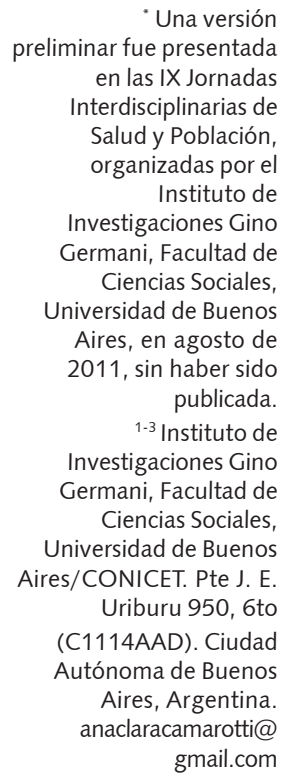

*Una versión preliminar fue presentada en las IX Jornadas Interdisciplinarias de Salud y Población, organizadas por el Instituto de Investigaciones Gino Germani, Facultad de Ciencias Sociales, Universidad de Buenos Aires, en agosto de 2011, sin haber sido publicada.

1-3 Instituto de Investigaciones Gino Germani, Facultad de Ciencias Sociales, Universidad de Buenos Aires/CONICET. Pte J. E. Uriburu 950, 6to (C1114AAD). Ciudad Autónoma de Buenos Aires, Argentina. anaclaracamarotti@ gmail.com 


\section{Resultados}

\section{Modelo negativizante-unidimensional}

A partir del análisis de los discursos de los docentes en los foros del curso virtual identificamos cuatro categorías emergentes que caracterizan a este modelo:

\section{La droga es un flagelo que castiga a los jóvenes aprovechando sus carencias y vulnerabilidades}

En esta categoría se observan ideas en torno a concepciones de los consumos de drogas que entienden a los jóvenes como sujetos carentes e incompletos. Entre los discursos de este grupo de docentes encontramos una postura estigmatizante de los jóvenes y en algunos casos también discriminatoria: "Ios jóvenes que se drogan son peligrosos, no aprecian sus vidas ni las de los otros", "cada vez más jóvenes se drogan", "la drogadicción es un paso a la delincuencia". Desde una visión pesimista, plantean la situación de consumo de drogas como algo que ya está instalado en la sociedad, como un hecho consumado y que poco o nada puede hacerse para erradicarla.

Esta posición discursiva puede vincularse con el Modelo de Creencias de Salud (Becker, 1974), que sostiene que si las personas poseen información sobre la severidad de las enfermedades, adoptarán conductas saludables si perciben que las conductas recomendadas son efectivas. Se parte del supuesto de que guiamos nuestras prácticas por aspectos racionales, que si tenemos información adecuada y creíble, adoptaremos las medidas de prevención necesarias.

El modelo explica las causas del consumo de drogas con teorías parciales, basadas en factores unidimensionales y específicos al individuo. Determinados rasgos de personalidad, ciertos comportamientos o carencias en la relación entre el individuo y el contexto social, eran algunas de las variables que entendía como determinantes al momento de consumir o no drogas. Para esta corriente, las sustancias no cumplen ninguna función (ni social, ni política, ni económica) en las sociedades, por lo cual le resulta muy difícil explicar por qué los consumos de drogas perduran, se diversifican e incrementan sus niveles.

\section{La droga "se filtra" en la escuela contaminando la vida de los jóvenes}

En estos discursos un aspecto relevante es la homogeneidad del concepto de drogas. El término es utilizado en singular y siempre se piensa que se trata de un consumo problemático. "La droga" aparece como algo ajeno, que se puede evitar y que es posible erradicar. Para ello es fundamental no probarla. Se subjetiviza a la droga (objeto) y se objetiviza y se cosifica al sujeto que consume, se lo persigue, critica, excluye, pero no se proponen alternativas posibles en relación a formas de prevención, a estrategias de contención, a tipos de tratamiento, etc.

Los docentes descontextualizan así el consumo, a la vez que no incluyen en sus explicaciones las relaciones que se establecen entre el tipo de sustancia/s que se consumen; la frecuencia de su uso; la trayectoria de la persona en el consumo de drogas; el perfil del consumidor; la edad de inicio en el consumo; el contexto social, cultural, económico en el que se encuentra la persona que está consumiendo.

Los discursos docentes que se agrupan en este modelo presentan un tono fatalista, trágico, moralizante. Se basan en ejemplos puntuales que son tan impactantes para ellos que hacen que el resto de los jóvenes, que probablemente tengan otros intereses, se diviertan con sus pares, trabajen, estudien, colaboren en sus casas, estén preocupados pensando en su futuro, pasen a segundo plano.

En este modelo se toman en cuenta sólo las denominadas "drogas ilegales"; no se hace mención en cambio al consumo de tabaco ni al de alcohol.

Los docentes alertan sobre el vacío institucional en el que viven (salud, educación, familia), fundamentalmente en lo que se refiere a la falta de procedimientos para abordar en la escuela situaciones concretas de jóvenes con problemas de consumos de drogas o adictos. Sin tener en cuenta 
que estos casos son poco frecuentes en la escuela, el hecho de no contar con procedimientos claros y estructurados colabora para que los docentes magnifiquen estas situaciones $y_{1}$ como consecuencia, se genera una falta de interés al momento de tener que encarar actividades de prevención, ya que consideran que abrir un espacio de diálogo más horizontal con sus alumnos puede terminar desbordándolos y/o agregándoles más trabajo.

Utilizar el término "flagelo" les permite desvincularse y externalizar un problema que los "desborda". Es en este sentido que entienden su trabajo individual como insuficiente porque frente a una problemática tan grandilocuente se requiere de todo un sistema especializado para atender dicha situación. Terminan entonces esperando recetas mágicas, externas y universales.

\section{La escuela debe dar la batalla en contra de la drogadicción}

Para este grupo de docentes la escuela es vista como la principal institución estatal "civilizatoria", encargada de encabezar la batalla contra el "flagelo de la droga" en nombre de las familias y de la sociedad en su conjunto. La función central de las instituciones educativas sería asegurar "un futuro a todos los jóvenes del país", dándoles herramientas para afrontar los riesgos generados por el mercado de las drogas y por las diversas situaciones de vulnerabilidad que viven los adolescentes.

En esta categoría encontramos un corrimiento en las interpretaciones que los docentes hacen del modelo más tradicional que caracterizamos en la primera categoría. El proceso de socialización se ubica como esquema explicativo de diferentes tipos de conductas problemáticas. Así, las conductas de las personas dependen de los vínculos que se establezcan con sus entornos de socialización; cuando el individuo adquiera una fuerte vinculación con ámbitos sociales y culturales será una persona integrada; cuando esto no funcione "correctamente" estaremos en presencia de sujetos "problemáticos", que en el caso de que consuman drogas pueden categorizarse como "desviados", "enfermos", "carentes".

Para estos docentes la escuela se ubica como el lugar fundamental para la "detección" y la derivación de los jóvenes que tienen problemas con el consumo de drogas. Si bien la escuela parece ser la protagonista en cuanto a dar soluciones a esta problemática, no termina siendo de este modo. Ella sólo se constituye como una intermediaria entre los jóvenes y las instituciones "especializadas": centros de prevención de adicciones, comunidades terapéuticas, psicólogos, centros de salud.

En ninguna de las posiciones anteriores se entiende a la escuela como una institución que pueda propiciar cambios concretos en estas problemáticas, con perspectivas de trabajo que utilicen e integren lo lúdico, lo expresivo, involucrando a todos los actores escolares (directivos, docentes, preceptores, alumnos) y de la comunidad en acciones de promoción de la salud que pongan en el centro los distintos saberes y experiencias de los sujetos.

\section{Los adultos son los principales responsables de formar y orientar a los jóvenes para evitar que caigan en la droga}

Para este grupo de docentes, los padres son los únicos responsables adultos del consumo de drogas de los jóvenes. Nunca emergen discursos críticos en torno a los modos que tienen docentes y directivos, como parte del colectivo de adultos, de relacionarse con los alumnos, lo que no contribuye hacia una mirada reflexiva acerca de qué cosas podrían modificar desde su práctica concreta para poder contribuir a algún cambio al respecto. De este modo, se externaliza el problema y la escuela sólo puede contribuir con la "detección" de los jóvenes que consumen.

Se parte de la idea de que los sujetos adultos lo son porque han alcanzado la madurez necesaria y por tanto nada tienen para aprender. Los jóvenes, en cambio, deben transitar este camino hacia la adultez para poder ser sujetos plenos. En ese recorrido las drogas se encuentran al asecho y los adultos, en el mejor de los casos, sólo pueden estar ahí para alertar a los jóvenes y no permitirles que "caigan" en el consumo. Es decir, el consumo de drogas aparece como una práctica exclusiva del colectivo de jóvenes; los adultos, en cambio, no tienen ningún riesgo ya que permanecen ajenos al mismo.

Desde el paradigma moralista - actualmente dominante en los abordajes que articulan educación y salud -, se reproduce una concepción de salud definida y reglada por los discursos biomédicos, 
orientada hacia la enfermedad como un problema individual. Esto bloquea la consideración de las dimensiones socio-políticas y de las condiciones estructurales en las que se enmarcan los problemas de salud, lo que los aleja de las definiciones más complejas, socio-políticas y globales del bienestar individual y colectivo, que fueron surgiendo a partir de las diversas críticas y experiencias desarrolladas desde la medicina social y que fueron parcialmente plasmadas en los documentos de la Organización Mundial de la Salud (OMS, 1998).

Desde esta postura las acciones de educación para la salud buscan imponer concepciones racionales del bienestar y de las conductas saludables, sin tener en cuenta las definiciones, experiencias y capacidades de reflexión sobre sí mismos de los sujetos implicados.

\section{Modelo reflexivo-multidimensional}

A partir del análisis de los discursos de docentes en el foro identificamos las siguientes cuatro categorías emergentes que caracterizan a este modelo:

\section{Es necesario realizar una ruptura con las concepciones unidimensionales de los consumos de drogas que parten de la negativización de los jóvenes}

Retomando las consignas y contenidos del curso, en los foros algunos docentes realizan una crítica a las concepciones estereotipadas en torno a los consumos de drogas. Frente a los discursos del modelo negativizanteunidimensional (identificados principalmente en los medios de comunicación masiva), que homogeneizan el fenómeno a partir de concepciones binarias y naturalizadas, estos docentes señalan su carácter heterogéneo y complejo. Identifican una pluralidad de prácticas de consumo de sustancias, atravesadas por dimensiones culturales, generacionales y/o de género que las van clasificando como legítimas/legales o ilegítimas/ilegales.

Desde esta mirada, los docentes critican a las posiciones discursivas de la patología social que, a partir de la homogeneización, establecen relaciones causales lineales entre los términos pobreza, droga, delito y juventud ${ }^{4}$. Algunos docentes señalan que estos discursos atraviesan la cotidianeidad de sus comunidades educativas, contribuyendo a procesos de estigmatización y/o expulsión de muchos estudiantes y de sus familias.

Este tipo de clima social escolar desubjetivante, que Di Leo (2009) analiza e identifica como dominante en escuelas secundarias públicas de la Ciudad Autónoma de Buenos Aires, contribuye a la crisis de sentido de las instituciones educativas y a la reproducción de concepciones reificadas de las subjetividades. La percepción y vinculación de los agentes escolares con los otros especialmente los jóvenes - se basa en la negación de sus capacidades de agencia y/o reflexividades, tanto desde los discursos naturalistas y/o psicologistas - falta de maduración - como desde su victimización social - objeto pasivo de las transformaciones económico-sociales y culturales. Se negativizan (es decir, se juzgan negativamente) las prácticas de los adolescentes, asociándolas fundamentalmente a las violencias, transgresiones $\mathrm{y} / \mathrm{o}$ riesgos sociales consumo de drogas, delito, ITS, VIH/sida, etc.

La percepción de una profunda brecha entre el mundo de los adolescentes visto como no reflexivo, incivilizado, violento - y el de los adultos - visto como reflexivo, civilizado -, genera en la mayoría de los docentes y directivos

\footnotetext{
${ }^{4}$ Tal como analiza Mariana Chaves (2005), desde la formación discursiva de la patología social la juventud es definida como el sector de la sociedad que está enfermo o es el más vulnerable para enfermarse. El adolescente es visto como un síntoma, portador de las consecuencias negativas de los cambios sociales (socioeconómicos, familiares, culturales, etc.). Esto constituye una mirada negativa, fuertemente asociada a diversos problemas y/o patologías sociales: alcoholismo, sida, drogas, embarazo adolescente, violencia. Esta formación discursiva está plagada de términos médicos, psicológicos, judiciales y/o sociológicos tanto en torno a los diagnósticos de las diversas patologías - entre las que ocupan un lugar central los consumos de drogas como a los diversos tipos de estrategias para su tratamiento, prevención, control y/o contención.
} 
${ }^{5}$ El modelo de la promoción de la salud parte de la concepción compleja y multidimensional de salud impulsada desde mediados del siglo XX por la Organización Mundial de la Salud

(OMS) dirigiéndose a propiciar que los sujetos participen activamente en su crítica y redefinición.

A partir del proceso de desnaturalización y reconocimiento reflexivo de las determinantes socioestructurales y sus influencias sobre las prácticas y

representaciones

individuales, se busca desarrollar las

potencialidades de los sujetos para criticary transformar ambas

dimensiones (Kornblit,

2010; Czeresnia

Machado de Freitas, 2006). sensaciones de una creciente incapacidad para ejercer el rol para el cual fueron formados y, en general, de una crisis de la autoridad y de la institución escolar, las que a su vez profundizan sus sentimientos de malestar y estrés laboral. Las consecuencias de estos fenómenos en la práctica pedagógica - desgano, dificultades para controlar las propias reacciones y establecer una comunicación con estudiantes y pares - retroalimentan en un círculo vicioso los procesos de desubjetivación tanto de sí mismos - objetos de las circunstancias - como de los otros (Di Leo, 2009).

\section{Los sujetos y sus contextos grupales, culturales y/o sociales deben ser colocados en el centro de los abordajes de los consumos problemáticos de drogas}

Partiendo de la ruptura epistemológica analizada en la categoría anterior, algunos docentes plantean la necesidad de realizar un desplazamiento del centro en los abordajes de los consumos problemáticos de drogas desde las sustancias hacia los sujetos y sus contextos. Critican así las políticas mediáticas y sanitarias que ponen en un lugar predominante a "la droga", soslayando la multiplicidad de experiencias individuales y de procesos político-económicos en los que se enmarcan los consumos y/o adicciones a sustancias en nuestras sociedades.

Desde estas miradas es posible abordar en su complejidad las transformaciones en los niveles de consumo de alcohol de los jóvenes. Según Baigorri Agoiz y Chaves Carrillo (2006), el crecimiento de dichos consumos se enmarca en una tendencia de alcance global, compuesta por el trinomio ocio-mercado-dimisión parental/ estatal. Con el desarrollo de la sociedad industrial, se institucionalizó el tiempo dedicado al descanso. Luego, con los regímenes de Estado de bienestar se profundizó este fenómeno, ya que no había que dedicar todo el tiempo a generar ingresos y a la reproducción familiar; sin embargo, el tiempo de ocio también fue mercantilizado. En la industria del ocio juega un rol central el creciente poder de las multinacionales del alcohol, cuyas inversiones publicitarias y esponsorizaciones se dirigen hacia la población joven. Simultáneamente, se retroalimentan dos formas de dimisión: a) por un lado, el abandono por parte de las familias de algunas de sus tradicionales formas de control; b) la crisis del Estado social, que se viene profundizado en muchos países desde la última década del siglo XX, que ha conducido a la disminución de las políticas dirigidas a la creación de espacios públicos destinados a jóvenes (Camarotti, Di Leo, Adaszko, 2010).

Esta perspectiva se articula con las propuestas y estrategias de prevención específica y/o inespecífica de los consumos problemáticos de drogas enmarcadas en el modelo de promoción de la salud ${ }^{5}$. Desde hace varias décadas los programas de reducción de daños no buscan centrarse en el consumo como tal, sino en los daños individuales y sociales que el mismo trae aparejado. Estas estrategias no consisten solamente en acciones de cambios de jeringas o programas de sustitución de opiáceos, también garantizan el acceso de todos a la información y a la prevención, facilitan el contacto de los usuarios de drogas con las instituciones de salud y/o educativas e intentan evitar los costos sociales e individuales que acompañan a la penalización de los consumos de drogas (Hopenhayn, 2002; Touzé, Rossi, 1993;). Por ejemplo, cuando dichos programas se centran en el segmento poblacional que está utilizando sustancias psicoactivas como el paco, no se limitan a encarar problemas sanitarios, como la prevención de infecciones y de sobredosis, sino que encaran también la marginalización, la exclusión social, la criminalización y la estigmatización (Comité Científico Asesor, 2009). 
3 La escuela puede constituir un espacio público para jóvenes que viven en contextos de pobreza, discriminación, violencias y consumos problemáticos de drogas

Algunos docentes señalan a la escuela como el único espacio público en el que pueden participar muchos jóvenes que viven en contextos de vulnerabilidad. En muchas ocasiones, a pesar de habitar en barrios con necesidades básicas insatisfechas, atravesados casi cotidianamente por situaciones de violencias, discriminación y/o consumos problemáticos de drogas, muchos jóvenes siguen asistiendo a la escuela pública, viéndola como un ámbito en el que pueden encontrarse con otros sujetos y otras realidades.

Algunos docentes e instituciones educativas llevan adelante acciones de promoción de la salud y/o reducción de daños. Desarrollan proyectos en los que se propicia la participación de los distintos agentes de la comunidad educativa, partiendo de la multiplicidad de experiencias y visiones de los sujetos en torno a los consumos de drogas, construyendo e intercambiando conocimientos y desarrollando estrategias preventivas centradas en el cuidado y el reconocimiento de sí mismo y de los otros. Estas acciones de promoción de la salud en las escuelas propician el despliegue de la autonomía, en el sentido propuesto por Cornelius Castoriadis (2008). En un proceso dialéctico y político nunca cerrado, los sujetos individuales y colectivos van apropiándose reflexivamente de los saberes que necesitan y con ellos van resignificando $\mathrm{y} / \mathrm{o}$ transformando sus condiciones objetivas y subjetivas de vida. Por ende, este proceso de construcción de la autonomía - horizonte nunca alcanzable totalmente - sólo se va posibilitando a partir de la transformación simultánea de las instituciones que co-constituyen a los individuos en todos los momentos de su vida (Di Leo, 2009).

\section{El diálogo entre jóvenes y adultos es fundamental} para construir espacios escolares de reconocimiento y transformación

Algunos docentes hacen hincapié en la centralidad del diálogo entre jóvenes y adultos como un requisito fundamental para la generación de estrategias de promoción de la salud y/o prevención de los consumos problemáticos de drogas. Como se analizó en torno a las categorías anteriores, estos docentes consideran que sólo a partir del diálogo intergeneracional es posible superar, desde las prácticas pedagógicas cotidianas, las posiciones discursivas negativizante-unidimensional y de la patología social, que centran el problema de los consumos de drogas en los jóvenes (especialmente los pobres), sin escucharlos y negándoles el reconocimiento como sujetos reflexivos.

Estas reflexiones y propuestas se articulan con los análisis de Axel Honneth (1997) en torno al lugar que ocupan las luchas por el reconocimiento en los procesos de constitución de las subjetividades. Según este autor, para la adecuada comprensión de los principales modelos de reconocimiento es necesario concretizarlos, abordándolos en relación a las grandes formas de negación de reconocimiento que movilizan a los individuos en los heterogéneos contextos sociales que atraviesan en sus vidas cotidianas. A partir específicamente del reconocimiento ético-social, los sujetos logran el reconocimiento recíproco. Retomando a George Mead (1968), Honneth (1997) señala que a partir de esta modalidad de reconocimiento - a la que también denomina solidaridad -, las normas éticas desde las cuales los sujetos se reconocen recíprocamente en su especificidad individual están abiertas a un proceso de destradicionalización, perdiendo su carácter jerarquizante y prescriptivo.

En torno a espacios de diálogo intersubjetivo como los propuestos por algunos docentes, se abren nuevas posibilidades para el despliegue en la escuela del reconocimiento, superando las prácticas discursivas desubjetivantes y/o de la tolerancia, actualmente dominantes en las instituciones educativas. Así, a partir del diálogo entre jóvenes y adultos, se abre la posibilidad de una reconstrucción de la legitimidad de la escuela, constituyéndose en un espacio público, es decir, en un espacio de ejercicio y despliegue de la libertad, autonomía y reflexividades de los sujetos (Di Leo, 2009a). 


\section{Reflexiones finales}

Los modelos "negativizante-unidimensional" y "reflexivo-multidimensional", que hemos analizado a lo largo del trabajo, no se presentan de manera pura en las posturas de los docentes. En sus intervenciones en los foros encontramos posturas que representan parcialmente a ambos.

En relación con el primer modelo, lo que aparece como menos flexible es la concepción del consumo de drogas en los jóvenes signada por el dramatismo, la espectacularidad y la negación de que puedan existir consumos de drogas que no sean problemáticos. Dichas rigideces están acompañadas de una desconexión de este fenómeno con respecto a las dimensiones históricas, sociales, culturales y generacionales. Esta representación social del fenómeno conduce a la no implicación de los docentes y de la escuela en el abordaje de estos temas en el ámbito educativo.

Asimismo, las escasas iniciativas de abordaje de los consumos de drogas realizadas en el marco de este tipo de posiciones se centran únicamente en la transmisión de información como generadora de cambios en las actitudes y prácticas individuales. La limitada correspondencia que tiene la mayoría de dichas acciones con las problemáticas que pretenden afrontar se debe fundamentalmente a que profundizan la distancia entre, por un lado, los saberes y prácticas disciplinarias y moralizadoras hegemónicas en las instituciones educativas; $y$, por el otro, la pluralidad de experiencias, modalidades de socialización y de construcción identitaria de los jóvenes. Por ello, este tipo de prácticas y discursos, más que abrir espacios de encuentro con los estudiantes, los clausuran, constituyendo barreras para la institucionalización de prácticas de promoción de la salud en la escuela.

En contraposición con el anterior, el segundo modelo analizado parte de una desnaturalización de las representaciones en torno a los consumos de drogas y su asociación exclusiva con la juventud. En sus reflexiones los docentes tienen en cuenta las relaciones que se establecen entre los contextos socioculturales y económicos, el perfil de los sujetos y las características de las diversas sustancias. Por otro lado, es destacable la resignificación de la escuela como espacio público en el que es posible la generación de diálogo entre adultos y jóvenes, desde los cuales se construye el conocimiento y el reconocimiento.

A medida que se propicien desde las instituciones educativas las acciones de promoción de la salud centradas en este tipo de posición, se abren nuevas posibilidades para la construcción de un diálogo entre los diversos sujetos que participan cotidianamente en las mismas, que se orientará hacia sus horizontes de felicidad, incorporando las dimensiones éticas y estéticas de la existencia (Ayres, 2002). A partir del diálogo entre las diversas experiencias y saberes de jóvenes y adultos, se abren nuevas posibilidades para la reconstrucción de la legitimidad de la escuela, constituyéndose, en términos de Hannah Arendt (1993), en un espacio público, un espacio de ejercicio y despliegue de la libertad de los sujetos.

En relación con este modelo hay que tener en cuenta que las categorías que aparecen de él pueden haber estado influidas por los contenidos del curso. Por otra parte, aún cuando no contamos con estudios previos, es posible suponer que las posturas de los docentes se encuentran atravesadas por los debates actuales sobre el tema, que cuestionaron fuertemente las posturas más tradicionales en torno a él.

Si bien el modelo está presente en buena parte de los docentes que hicieron el curso, las propuestas que ellos formularon en relación con sus posibilidades de implementación son aún débiles, por lo que podría suponerse que hay barreras que obstaculizan la transformación de los contenidos teóricos incorporados en propuestas de intervención preventivas. Es posible que estas barreras se vinculen con las rigideces de la institución escolar y del habitus docente tradicional, cuya transformación requiere procesos de reflexión y capacitación de largo aliento que comiencen en la formación inicial. En muchos casos estas posiciones llevan al aislamiento de los docentes dispuestos a introducir cambios en las prácticas pedagógicas. Ello conduce a un desgaste personal y a reacciones y resistencias de parte de sus colegas. 


\section{Colaboradores}

Los autores trabajaran de manera conjunta en todas las etapas de producción del manuscrito.

\section{Referencias}

ARENDT, H. La condición humana. Buenos Aires: Paidós, 1993.

AYRES, J.R.C.M. Conceptos y prácticas en salud pública: algunas reflexiones. Rev. Facul. Nac. Salud Publ., v.20, n.2, p.67-82, 2002.

BAIGORRI AGOIZ, A.J.; CHAVES CARRILLO, M. Botellón: más que ruido, alcohol y drogas. Anduli: Rev. Andal. Cienc. Soc.,v.1, n.6, p.159-73, 2006.

BECKER, M. The health belief model and illness behavior - health education. Monographs, v.2, n.4, p.409-19, 1974.

CAMAROTTI, A.; DI LEO, P.; ADASZKO, D. Experiencias juveniles nocturnas, usos y significados en torno al consumo de alcohol en tres ciudades de Argentina. Rev. Cons. Prof.,v.1, n.45, p.14-5, 2010.

CASTORIADIS, C. Un mundo fragmentado. La Plata: Terramar, 2008.

CHAVES, M. Juventud negada y negativizada: representaciones y formaciones discursivas vigentes en la Argentina contemporánea. Ultima Decada, v.1, n.23, p.9-32, 2005.

COMITÉ CIENTÍFICO ASESOR EN MATERIA DE CONTROL DEL TRÁFICO ILÍCITO DE ESTUPEFACIENTES, SUSTANCIAS PSICOTRÓPICASY CRIMINALIDAD COMPLEJA SOBRE LOS USUARIOS DE DROGAS Y LAS POLÍTICAS PARA SU ABORDAJE, 2009. Disponible en: <http:// www.jgm.gov.ar/archivos/comisionnacional/DO1usuarios.pdf>. Acceso en: 15 mayo 2012.

CZERESNIA, D.; MACHADO DE FREITAS, C. (Orgs.). Promoción de la salud: conceptos, reflexiones y tendencias. Buenos Aires: Lugar, 2006.

DI LEO, P.F. La promoción de la salud como política de subjetividad: constitución, límites y potencialidades de su institucionalización en las escuelas. Salud Colect., v.5, n.3, p.377-89, 2009.

HONNETH, A. La lucha por el reconocimiento: por una gramática moral de los conflictos sociales. Barcelona: Crítica, 1997.

HOPENHAYN, M. (Comp.). Prevenir en drogas: enfoques integrales y contextos culturales para alimentar buenas prácticas. Santiago de Chile: Naciones Unidas, 2002. (CEPAL - Serie Políticas Sociales, 61).

KORNBLIT, A.L. La promoción de la salud entre los jóvenes. Acta Psiquiat. Psicol. Am. Lat., v.56, n.3, p.217-26, 2010.

MEAD, G. Espíritu, persona y sociedad. Buenos Aires: Paidós, 1968.

OMS. Promoción de la Salud. Glosario. Ginebra: OMS, 1998. Disponible en: <http://www.bvs.org.ar/pdf/glosario_sp.pdf>. Acceso en: 7 mar. 2013.

TOUZÉ, G.; ROSSI, D. Sida y drogas: ¿abstención o reducción del daño? Buenos Aires: FAT, 1993. 
Desde 2010 nuestro equipo diseña y coordina un curso virtual sobre Prevención del Consumo Problemático de Drogas dictado por el Ministerio de Educación de Argentina. En el momento cuenta con tres ediciones en las que participaron alrededor de 6.000 docentes. En este artículo buscamos: a) analizar los discursos de los participantes para identificar sus posiciones en referencia al consumo de drogas; b) reflexionar sobre las implicaciones de estas posiciones para el desarrollo de estrategias de promoción de la salud en la escuela. Para el análisis del corpus seguimos los lineamientos de la teoría fundamentada, identificando dos grandes posiciones discursivas: modelo negativizanteunidimensional y modelo reflexivo-multidimensional. Mientras que el primero conduce a la no implicación de los generación de espacios escolares de diálogo y de reconocimiento entre adultos y jóvenes.

Palabras clave: Instituciones académicas. Docentes. Adolescente. Educación a distancia. Discursos sobre uso de drogas.

Prevenção do consumo problemático de drogas na escola: estratégia de formação de professores na Argentina utilizando TIC

Desde 2010 nossa equipe projeta e coordena um curso virtual ditado pelo Ministério Nacional de Educação da Argentina. Até hoje, conta com três edições nas quais participaram cerca de seis mil professores de todo o país. Neste artigo procura-se: a) analisar o discurso dos professores para identificar as suas posições sobre o uso de drogas; b) refletir sobre as implicações dessas posições discursivas para o desenvolvimento de estratégias de promoção da saúde na escola. Para a análise do corpus, seguimos as diretrizes da teoria fundamentada, identificando duas grandes posições discursivas: modelo negativizante-unidimensional e modelo reflexivomultidimensional. Enquanto o primeiro leva ao descompromisso dos professores e da escola na abordagem dessas questões, o segundo propicia a geração de espaços escolares de diálogo e reconhecimento entre adultos e jovens.

Palavras-chave: Instituições acadêmicas. Docentes. Adolescentes. Educação a distância. Discursos sobre uso de drogas.

\section{Prevention of problematic drug use in schools: teacher training strategy in Argentina using ICT}

Starting in 2010, our team has designed and coordinated a virtual course on problematic drug use, for teachers, commissioned by the Ministry of Education in Argentina. So far, the course has been run three times, with participation from around 6.000 teachers across the country. In this article, it was sought: a) to analyze the teachers' discourse in order to identify their positions relating to drug use; b) to reflect on the implications of these discursive positions for development of health promotion strategies in schools. To analyze the corpus, we followed the guidelines of grounded theory and identified two major discursive positions: a negational single-dimensional model and a reflective multidimensional model. While the former leads to disengagement among teachers and their schools in addressing these issues, the latter aims to generate school spaces for dialogue and recognition between adults and adolescents.

Keywords: Schools. Faculty. Adolescent. Education distance. Speeches about drug use. 
